\title{
XXI. Mittheilungen aus dem mineralogischen Museum der Universität Bonn.
}

\author{
VI. Theil.
}

Von

H. Laspeyres und E. Kaiser in Bonn.

(Mit 3 Textfiguren.)

\section{Quarz- und Zirkonkrystalle im Meteoreisen von Toluca in Mexico*).}

Schon acht Jahre bevor Story-Maskelyne in dem Meteorit von Breitenbach in Böhmen den Asmanit (Tridymit) entdeckte ${ }^{*}$ ), hatte G. Rose Krystalle von Quarz im Meteoreisen von Toluca beobachtet***).

Er fand in der Sammlung des Geh. Sanitätsratbs Nagel in der Rostrinde eines Stúckes Eisens von Xiquipilco eingewachsen einen etwa $\frac{1}{3}$ Linie grossen glänzenden Krystall, der sich mit Hinterlassung eines glattflächigen Eindruckes aus dem Eisenrost nehmen liess und die Form, sowie die gut messbaren Winkel des Quarzes f) zeigte. Ein kleines Bruchstuck eines anderen daneben befindlichen Krystalles konnte Rose vor dem Löthrohre prufen.

Das war der erste bestimmt nachgewiesene Fund dieses Minerals in Meteoriten. Wohl hatte schon C. A. Joy im Lösungsruckstande des Meteoreisens von Cosby Greek ttj wie Quarz aussehende Körner gefunden, aber

*) Vorgelegt in der niederrheinischen Gesellschaft für Natur- und Heilkunde zu Bonn 12./11. 94. Kölnische Zeitung Nr. 1016.

**) Proceedings of the Royal Soc. of London 1869, 370-72; Philos. Transactions of the Royal Soc. of London 1871, 161, 359-365.

****) Monatsber. d. Akad. d. W. zu Berlin 1861, 406-409; Pogg. Ann. 1861, 113, 184-188, 1865, 124, 201 ; Beschreib. und Eintheil. der Meteoriten 1864, 42-43, 6062, 139 (a. d. Abhandl. d. Akad. d. W. zu Berlin 1863).

t) Gemessen wurde $\{10 \overline{1} 1\} R:\{01 \overline{1} 1\}-R$, Randkante $76^{\circ} 20^{\prime}-25^{\prime}$ und $76^{\circ} 11^{\prime}-$ 35'; Endkante $46^{0} 18^{\prime}-30^{\prime}$.

tf) Ann. d. Chem. u. Pharm. 4853, 86, 43. 
dieselben nicht näher untersucht, sondern nur nach ihrer Härte gegen Glas und Bergkrystall als Quarz angesprochen.

Rose bielt es sofort für wahrscheinlich, dass unter den von Wöhler, Pugh und Uricoechea beobachteten, in Säuren unauflöslichen Körnern in dem Meteoreisen von Toluca ebenfalls Quarz enthalten sei, verfolgte aber diese Vermuthung nicht weiter, sondern gab es Wöhler anheim, falls dieser noch im Besitze der erwähnten Körner wäre.

"Zwar könnte man Zweifel hegen - fäbrt Rose fort - ob der beschriebene Quarzkrystall wirklich dem Meteoreisen angehört hat und nicht vielmehr erst nach dem Falle, beim Liegen in und auf der Erde, und der dabei stattfindenden Oxydation seiner Oberfläche sich damit verbunden hat; indessen war der Krystall vollkommen von braunem Eisenoxyde umgeben und dieses verband sich so vollkommen mit dem unzersetzten Eisen, dass man dasselbe für nichts anderes als für die entstandene oxydirte Rinde, und den Quarz als darin ursprünglich eingewachsen annehmen kann. «

"Ausserdem sind doch Quarzkrystalle in dem Sande nur recht selten, und in dem Toluca-Thale ist Quarzsand vielleicht gar nicht vorhanden, da die umgebenden Berge aus quarzlosem Trachyt oder neueren vulkanischen Gesteinen bestehen «. "Bei den vielen Stücken, die sich durch G. A. Stein und Dr. Krantz in den Sammlungen finden, wird man vielleicht auch noch bei anderen Stücken Quarzkrystalle beobachten und die gemachte Entdeckung bestätigen können. “

G. Rose hatte aber ausserdem nur noch an einer Stufe dieses Eisens im Berliner mineralogischen Museum kleine quarzähnliche Körner an der Oberfläche sitzen sehen, doch gingen dieselben vor der Untersuchung verloren. Er hielt deshalb die Quarzkrystalle im Meteoreisen von Toluca fur selten.

Ist auch die Beweisfuhrung von G. Rose, der Quarzkrystall könne nicht von angerostetem Sande herrubren, nicht einwandfrei, so stimente doch G. vom Rath der Ansicht Rose's zunächst vollkommen zu*). Allein schon zwei Jahre später hat G. vom Rath diese Ansicht geändert.

Bei seiner Bearbeitung der hiesigen Meteoriten fand er nämlich drei von Xiquipilco mit "Quarzkrystallen in der oxydirten Rinde (**).

"Auf der Oberfläche - so schreibt er - haften scheinbar eingewachsen zahlreiche kleine Quarzkrystalle, sich stellen weise zu einem Aggregat verbindend. Diese Quarze sind indess dem Meteoreisen fremd und lediglich durch Oxydation des Eisens oxydirt***) worden; sie ruhren von

*) Verhandl. d. naturhist.' Ver. pr. Rheinl. u. Westf. 1873, 30, 108, Sitzungsber.

**) Daselbst 1875, 32, 360-361, Nr. 4 f., $4 q$ u. $4 h$.

***) „oxydirt a ist wohl Druckfehler. 
dem Quarzsande her, in welchem die kosmischen Körper lange gelegen. In der Weise ist die frühere Angabe des Vorkommens von Quarz im Meteoreisen von Toluca zu deuten. "

Hierauf berubt wohl die gleichlautende Meinung von L. Fletch er ${ }^{*}$ ) und ganz neuerdings auch dieselbe Annahme von Seiten E. Gohen's**), obgleich gerade Letzterer durch seine mikroskopischen Untersuchungen der Lösungsrückstände mehrerer Meteoreisen dazu beigetragen hat, das bis dahin nur wahrscheinliche Vorkommen von mikroskopisch kleinen Quarzkörnern in dem Meteoreisen über die seitherigen Zweifel zu erheben und deren grössere Verbreitung wahrscheinlich zu machen $\left.{ }^{* * *}\right)$.

Bei dieser Sachlage und bei der reichlichen Menge von solchem Quarz an einem dieser Meteoriten in der hiesigen Sammlung schien mir der Versuch lohnend, diese Streitfrage zwischen so bedeutenden Mineralogen und Meteoritenkennern zu lösen.

Diese Stufe, eine der grössten der von Krantz 1856 im Toluca-Thale gesammelten Eisenmassen, $10030 \mathrm{~g}$ schwer, zeigt auf der gerosteten Oberfläche mehrere ganz unregelmässig oder schlierenartig begrenzte, bis $50 \mathrm{~mm}$ lange und breile, aber immer nur wenige Millimeter dicke erdige Partien, die auf den ersten Blick allerdings völlig einem durch Rost angehefteted, äusserst feinen und etwas lehmigen Sande gleichen. In diesem fast dichten Teige liegen eingewachsen zahlreiche, bis $2 \mathrm{~mm}$ grosse, lebhaft glänzende Quarzkrystalle, wie sie G. Rose beschrieben hat.

Zugleich erinnern auch solche Partien an manche ganz zersetzte Olivinknollen der Meteoriten und Basalte, nur dass diesen die Quarze fehlen.

Die Quarzkrystalle schälen sich, wie es $\mathrm{R}$ os e beschrieben hat, schon mechanisch leicht aus dem feinsandigen Teige heraus, noch schöner erhält man die wasserklaren Bergkrystalle von der Form $\{10 T 0\} \infty R,\{10 T 1\} R$, $\{01 T 1\}-R$ durch Lösen des Teiges in Salzsäure.

Wie alle Silicate der Meteoriten sind auch diese Quarze ungemein rissig und spröde, so dass sie leicht in unregelmässig begrenzte Splitler beim Herausscbälen oder Lösen zerfallen.

Die besten Reflexe im Fernrohrgoniometer geben die ungestreiften Rhomboëderflächen

$$
\begin{aligned}
& (10 \bar{T}) R:(10 \bar{T})-R \quad 46^{0} 5^{\prime}-8^{\prime} \quad\left(\text { ber. } 45^{0} 16^{\prime}\right) \\
& (10 \bar{T}) R:(10 \bar{T} 0) \infty R \quad 3744-47 \quad(-3813) .
\end{aligned}
$$

Weder mit Lupe, noch unter dem Mikroskope konnte an den Krystallen

*) An Introduclion to the study of Meteorites; British Museum (Natural History) Mineral. Department 1890, 32.

**) Meleoritenkunde 1894, 1, 215-216, 323.

***) Annalen d. k. k. naturhist. Hofmuseum in Wien $1891,6,140,145,151,156$, $159 ; 1892,7,152$. 
auch nur eine Spur von einer matten sandkornartigen Abrollung der Kanten und Ecken oder einer Schrammung der Flächen bemerkt werden.

Unter dem Mikroskope erweist sich die Krystallform ganz ähnlich wie die aller in dem Meteoreisen eingewachsenen Krystalle, namentlich der bekannten Olivine im Pallaseisen, glänzend gerundet an den Kanten und Ecken mit "gleichsam angedrückten oder angeschliffenen spiegelnden Krystallflächen *.

Der den Quarz einbettende Teig wurde mit Salzsäure und darauf mit Natriumcarbonatlösung ausgekocht. In Lösung ging Eisen (Eisenrost), etwas Thonerde (Plagioklas oder daraus entstandener Thon), etwas Magnesia (Olivin) und Kieselsäure. Zuruck blieb ein áusserst feiner, scheinbar sandiger Rückstand.

In Canadabalsam eingebettet wurde derselbe der mikroskopischen Untersuchung unterzogen.

Da zeigte sich denn sofort wieder, dass von einem angebackenen Sande nicht die Rede sein kann, denn nirgends zeigt sich eine Spur von Abrollung. Der ganze Rückstand besteht nur aus scharfkantigen und scharfeckigen Körnern, breitsäulenförmigen Splittern und scharf ausgebildeten Krystallen.

Noch mebr als seine Form spricht der mineralische Bestand des Rückstandes gegen die Annahme von Sand.

Der grössere Theil, etwa $60 \%$ des Rückstandes, sind farblose wasserklare Körner, die nicht nur dem Quarze, sondern auch dem Plagioklas (nicht Anorthit, weil unlöslich in Salzsäure) und vielleicht auch dem Orthoklas angehören.

Letztere zeigen nämlich neben der guten Spaltbarkeit keine Zwillingslamellen, während solche beim Plagioklas daneben so zahlreich, oft auch scharf begrenzt und zum Theil auch gebogen wie in den irdischen Gesteinen sich zeigen, manchmal sogar in gewöhnlichem Lichte*).

Neben diesen völlig farblosen Splittern finden sich einzeln ganz schwach röthliche, gleichfalls klare, ab und zu mit Spuren von Spaltbarkeit, isotrop, stärker als Quarz und Glas das Licht brechend, aber viel schwächer als der gleichfalls röthliche Zirkon. Ich kann diese Splitter nur für Granat ansprechen $\left.{ }^{* *}\right)$.

*) Orthoklas in den Meteoriten ist übrigens schon von Anderen angegeben worden. E. Cohe n (Meteoritenkunde $1894,1,33,311$ ) hält aber diese Angaben für durchaus zweifelhaft.

**) Auch von anderer Seite sind Granat oder granatähnliche Körner in den Meteoriten angegeben worden, sicher erwiesen ist deren Vorkommen aber noch nicht.

C. U. Shepard; American Journal of Sciences 1846, 2, 380-382; 1848, 6, 407; $1857,24,136$.

C. W. Güm b el, Sitzungsber. d. Akad. zu München 1875, 323.

E. Cohen, Ann. d. k. k. naturhist. Hofmuseum in Wien 1894. 6, 141-159; Meteoritenkunde $1894,1,33,319,323$. 
Glaskörnchen, wie solche von E. Gohen und Weinschenk beobachtet worden sind $\left.{ }^{*}\right)$, konnte ich dazwischen nicht auffinden.

Der kleinere Theil, etwa $4.0 \%$ des Rutckstandes, besteht aus einem braungrünen oder grünen, je nach der Dicke bald helleren, bald dunkleren Minerale in zum Theil unregelmässigen, zum Theil breitsäulenförmigen Splittern mit rechteckiger Umgrenzung, deren Längsrichtung eine recht vollkommene prismatische Spaltbarkeit folgt. Fällt auch in diesen Spaltungsstiicken die Schwingungsrichtung des Lichtes manchmal mil den Spaltungstracen zusammen und zeigt sich auch in manchen Lamellen deutlich Pleochroismus, so gebören doch wohl alle dem monoklinen Augit an, denn bei manchen pleochroitischen Splittern bildet die Schwingungsrichtung mit den Spaltungstracen einen schiefen Winkel, und Hornblende ist bisher noch nicht in den Meteoriten beobachtet worden. Querbruche bekommt man unter dem Mikroskope nicht zu sehen.

Der interessanteste Gemengtheil des Rückstandes sind nun aber 0,04 bis $0,15 \mathrm{~mm}$ lange und 0,02 bis $0,07 \mathrm{~mm}$ dicke ungemein scharf und flächenreich ausgebildete Krystalle, welche nach Form und optischem Verhalten nichts anderes sein können als Zirkon, der allerdings bisher noch aus keinem Meteoriten angegeben wird**).

Diese gar nicht seltenen Krystalle zeigen stets dieselbe, in den beiden nebenstehenden Figuren dargestellte, nach der Hauptaxe prismatische tetragonale Krystallform :

$$
\begin{array}{ll}
o=\{111\} P & q=\{110\} \infty P \\
p=\{100\} \infty P \infty & d=\{m 11\} m P m . \\
o^{\prime}=\{101\} P \infty &
\end{array}
$$

Fig. 1.
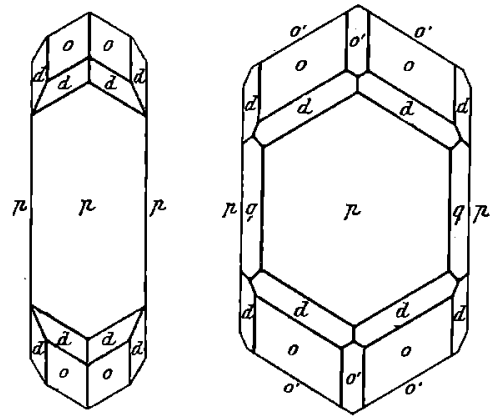

*) Annal. des k. k. naturhist. Hofmuseum in Wien 1891, 6, 141, 151, 159; 1892 , 7, 152; Meteoritenkunde 1894, 1, 317.

**) E. Cohen, Meteoritenkunde 1894, 1, 323.

Nach Abfassung dieser Mittheilung macht mich E. Cohen, dem ich ein Präparat dieses Rückstandes mit einem Zirkonkrystalle geschickt hatte, brieflich (21./12. 94) darauf aufmerksam, dass er in seiner inzwischen erschienenen III. Meteoreisenstudie (Ann. d. k. k. naturbist. Hofmuseum in Wien 1894, 9, 112) im Lösungsrückstande des Meteoreisens von Rasgata, Columbien, einen "säulenförmigen Krystall mit pyramidaler Endigung und paralleler Auslöschung, den gewöbnlichen Zirkonmikrolithen gleichend a beobachtet habe. In seinem Briefe bemerkt E. Coben dazu, dass er solchen Zirkonkrystall, dem von mir gesand ten durchaus gleichend, aber nicht so scharfe Formen zeigend, nur e in mal beobachtet habe. Er habe denselben in seiner Meteoritenkunde nicht erwabnt, weil er erst weitere Vorkommnisse abwarten wollte, da es ihm nicht ausgeschlossen erschien, dass sich ein solches Kryställchen aus dem Schleifmateriale beim Scbneiden des Meteoreisens in dasselbe eingepresst haben könnte. 
Bald sind sie lang und dünn, bald kurz und dick.

Stets sind die Krystalle an beiden Enden gleichmässig ausgebildet und zeigen keine Spur von sandiger Abrollung. Der ebene Scheitelwinkel zwischen zwei in derselben Axenebene liegenden Endkanten von $\{111\} P$ konnte vielfach unter dem Mikroskope gemessen werden, im Mittel zu $114^{\circ} 30^{\prime}$ (berechnet beim Zirkon $114^{\circ} 43^{\prime}$ ).

Spaltbarkeit, wie am Rutil, konnte nirgends beobachtet werden, einmal aber schöner muscheliger Bruch àn einer Kante des Prismas.

Die immer licht rölhlichen, ganz klaren und meist reinen Kryställchen zeigen ein sehr hohes Brechungsvermögen, sehr lebhafte Interferenzfarben, und die Axe der kleinsten optischen Elasticităt fällt mit der Hauptaxe zusammen.

Sie gleichen vollständig den mikroskopischen Zirkonkrystallen in den irdischen Gesteinen *) auch in Betreff mancher ihrer Einschlusse (s. u.).

Einzelne schwarze, krystallinisch scharf umgrenzte Körner im Lösungsrückstande sind vielleicht Ghromit oder Cliftonit, schwarze wohlumgrenzte Tafeln vielleicht Graphit. Dazu kommen nun noch als Bestandtheile des vermeintlichen Sandes die in Salzsäure schon gelösten Olivin und Anorthit.

In allen durchsichtigen Bestandtheilen des Lösungsrückstandes, am bäufigsten allerdings im Quarz, finden sich dunkel umrandete Gasporen und ungleich schmaler umgrenzte Einschlusse eines Mittels von dem Brechungsvermögen etwa des Glases oder einer Flüssigkeit. Immer' enthalten dieselben kugelrunde Gasblasen, meist einzeln, aber auch zu zwei, zu drei, ja zu vier wurden sie beobachtet. Weder von selbst, noch durch Erschutterung, noch durch Erwärmung bis zum Erweichen des Canadabalsam - also uber $32^{\circ} \mathrm{G}$. - ändert sich die Lage oder Grösse dieser Gasblasen. Sie liegen demnach wohl in Glaseinschlüssen, welche sehr hăufig in den Meteoriten sizd, während noch niemals ein Flussigkeitseinschluss darin ermittelt werden konnte $\left.{ }^{* *}\right)$. Schliesslich beobachtet man in a lle n Bestandtheilen des Lösungsrúckstandes, ganz besonders häufig allerdings im vermeintlichen Orthoklas, auch im Zirkon, winzige doppeltbrechende Krystallnadeln genau von dem Aussehen wie die A pa titnädelchen in den irdischen Gesteinen.

Allerdings ist bisher Apatit noch in keinem Meteoriten mit Sicherheit nacbgewiesen worden***), bekannt ist aber, dass Rammelsberg †) im

*) K. v. Chrustsch of f, Min. und petrogr. Mittheilungen von G. T s chermak 1886, 7, 423-443, Tf. 8.

**) E. Cohen, Meteoritenkunde 1894, 1, 326, 329.

***) E. Co b e n, Meteoritenkunde 1894, 1, 33, 323.

t) Chemische Natur der Meteoriten; Abhandl. d. Akad. d. W. zu Berlin 1870, 130 ; Pogg. Ann. 1848, 73, 585; Handb. d. Mineralchemie 1860, 937. 
Eukrit von Juvinas 0,28\% Phosphorsäure gefunden hat, die 0,60\% Apatit entsprechen würde. Da nun dieser Eukrit fast gar kein Nickeleisen enthält, das der Träger von Phosphor sein könnte, so wird das Vorkommen von Apatit in den Meteoriten auch durch diese chemische Beobachtung wahrscheinlich gemacht.

Lassen hiernach Form und mineralischer Bestand des Lösungsrückstandes keinen Zweifel, dass derselbe kein fremder Anschluss, sondern ein ursprünglicher Bestandtheil des Meteoreisens sein könne, so hielt ich es doch für erwünscht, mit diesen Ergebnissen die steinigen Lösungsrúckstände des Toluca-Eisens zu vergleichen.

Diese sind schon durch E. Cohen und Wein schenk*) mikroskopisch und mikrochemisch untersucht worden. Ihre Ergebnisse stimmen nicht völlig mit den meinigen uberein.

Sie fanden nämlich:

1. farblose, wasserklare Quarzkörner mit Gasporen, vielleicht auch mit Glaseinschlüssen, einige Male auch mit winzigen Mikrolithen;

2. farblose, wasserklare Körner mit schwacher Einwirkung auf das polarisirte Licht;

3. mattweisse Körner, einem durch Säure stark angegriffenen Silicate gleichend $\left.{ }^{* *}\right)$;

4. Cliftonit;

5. Chromit;

6. Cordierit-ähnliches Mineral, d. h. zwei dicksäulenförmige Körner mit lebhaften Interferenzfarben und Glas?-Einschlüssen, fleckig blau (pleochroitisch) und farblos;

7. isotrope, licht fleischrothe Granat-ähnliche Körner;

8. dunkelbraune, isotrope Körner mit starkem Glanze;

9. wasserklare, isotrope Glassplitter, vielleicht von den Glasgefässen herruhrend;

10. lichtgrüne, unvollkommen säulenförmig begrenzte, „schief auslöschende “ Augit-ähnliche Körner;

11. lichtgrünliche, säulenförmige, vollkommen spaltbare Splitter mit "gerader Auslöschung " und schwachem Pleochroismus, vielleicht rhombischer Pyroxen.

Die unter 2, 6, 8, 9 aufgefựhrten Körner habe ich in meinen Präparaten nicht beobachtet.

Wesentliche Abweichungen zwischen den beiderseitigen Beobachtungen sind mithin allerdings nur die Nichtangabe der Feldspäthe, des Zirkon und des Apatit, wobei nicht ausser Acht gelassen werden darf, dass die

*) Annal. d. k. k. naturhist. Hofmuseum in Wien 1891, 6, 140.

**) Nach meinen Beobachtungen der Kieselsäurerückstand der zersetzten olivine. 
unter Nr. 1 genannten Mikrolithen meinen für Apatit angesprochenen Krystallnadeln und die unter Nr. 2 aufgeführten Körner den von mir als Orthoklas gedeuteten Körnern entsprechen könnten.

Wegen dieser Abweichungen löste ich zunächst eine grössere Menge der im Laufe der Jahre im feuchten Museum aus dem Eisen gebildeten und von dem Eisen blätterig abgerosteten Rinde und erhielt hierbei genau dieselbe Lösung und den völlig gleichen Ruckstand wie bei jenen vermeintlich "sandigen Partien " von der Oberfläche, nur in sehr geringer Menge.

Um dem immerhin möglichen Einwande zu begegnen, diese Rindentheile hätten sich schon auf der natürlichen Lagerstätte gebildet und wären mit Sand untermischt worden, kochte ich in Salzsäure ein derbes festes, aber schon meist in Brauneisenstein umgewandeltes $82 \mathrm{~g}$ schweres Stück Toluca-Eisen aus der Krantz'schen Sammlung, welches von zahlreichen noch frischen Tänitlamellen regelmässig durcbsetzt war. Nachdem sich etwa die Hälfte gelöst hatte, zeigte der übrig gebliebene Kern mehrere ungefähr parallel laufende dünne Schlieren von hellgrauen körnigen zersetzten Silicaten bezw. abgeschiedener Kieselsäure, die sich in Natronlauge löste.

Diese Schlieren hatten ganz die Gestalt und Zusammensetzung jener oberflächlichen steinigen Partien. In Lösung gingen wieder Magnesia, Eisen, Thonerde, Kalkerde, mithin vorherrschend Olivin und etwas Anorthit; der Lösungsrückstand zeigle unter dem Mikroskope genau dieselben Bestandtheile wie in den beiden früheren Fällen.

v. Reichenbach*) hat schon mitten im Toluca-Eisen als gar nicht so selten ziemlich grosse Schlieren von steiniger Beschaffenheit, die er fur Olivin hielt, beschrieben und abgebildet.

Aehnliche Silicateinstreuungen sind auch in anderen Meteoreisen schon beobachtet worden ${ }^{* *}$ ).

Es unterliegt somit keinem Zweifel, dass Quarz und Zirkon, sowie die übrigen damit vorkommenden Gemengtheile ursprüngliche Bestandtheile des Toluca-Eisens sind.

Vielleicht gelingt es mir, durch die schon in Angriff genommene Auflösung grosser Mengen der im Museum nach und nach von den grossen

*) Pogg. Ann. 1857, 101, 311; $1857,102,618,621$.

Vergl, auch Uricoechea, Anv. d. Chem. u. Pharm. 1854, 91, 249-252.

Pugh, Ann. d. Chen. u. Pharm. 1856, 98, 383-386; Journ. f. prakt. Chem. 1856, $69,309-310$.

Wöh le r, Sitzungsber. d. Wiener Akad., malh.-nalurw. Cl. 1852, 8, 502.

**) z. B. Rasgata: W ö hl er, Sitzungsber. d. Wiener Akad., math.-naturw. Cl. 1852, 8, 502 .

Magura. Weinschenk, Cohen, Brezina, Annalen d. k. k. naturhist. Hofmuseum in Wien 1889, 4, 93-110; 1890, 5, 112-114; $1891,6,149-152,165$. 
Blöcken des Toluca-Eisens abgeblätterten Rostrinde soviel Lösungsrückstand zu erbalten, um auf chemischem Wege darin den Zirkon zu bestätigen und den vermutheten Orthoklas und Apatit sicher nachzuweisen.

\section{Chemische Zusammensetzung des Meteoreisens von Werchne Udinsk in Sibirien.}

Von diesem seltenen und deshalb nur spärlich in den meisten Sammlungen vertretenen, 1854 gefundenen Meteoreisen giebt es bisher nur eine, wenig genaue Analyse von v. Kotschubei*).

Die hiesige Meteoritensammlung gestattete mir, ein 2,3547 g schweres Sttuck desselben näher zu untersuchen. Um möglichst die mittlere Zusammensetzung des Eisens zu erhalten, wurde das ganze Stück aufgelöst.

Nach dem sorgfältigen Reinigen mittelst scharfer Bürste mit Alkohol und Aether (zur Entfernung etwa anhaftender Schneid- und Schleifmittel) wurde das ganz rein metallische Stuck nach der von E. Goh e $n^{* *}$ ) angegebenen Methode binnen fünf Wochen in kalter, ganz verdünnter Salzsäure gelöst.

Die hierbei sich entwickelnden Gase durchstrichen eine Silberlösung, aus der nur unbestimmbare Mengen von Schwefelsilber ausgefällt wurden. Das angewandte Stuck Meteoreisen enthielt mithin nur Spuren von Schwefeleisen (Troilit).

Der in so schwacher Salzsäure unlösliche Rückstand bestand aus schwarzen, kohligen, unter dem Mikroskope graphitartigen, zum grössten Theile verbrennbaren Schuppen, aus Silicatkörnchen und aus den in starker und warmer Salzsäure löslichen Blättchen und Nädelchen von Phosphornickeleisen (Schreibersit, Rhabdit) mit messinggelben Anlauffarben.

Unter dem Mikroskope näher untersucht bestehen die unlöslichen Silicate aus winzigen, unregelmässig begrenzten, farblosen, stets doppeltbrechenden Splittern, die ihrer Art nach nicht mit Sicherheit zu erkennen sind. Am meisten gleichen sie theils dem Quarz, theils dem farblosen Bronzit (Enstatit).

In der salzsauren Lösung konnten nur Phosphor, Kupfer, Magnesium***), Nickel und Kobalt neben dem Eisen nachgewiesen werden.

Auf die genaue Bestimmung von Nickel und Kobalt wurde die grösste Sorgfalt verwendet. Die geschiedenen Metalle erwiesen sich völlig rein.

*) G. Rose, Beschreib. u. Einth. d. Met. (Abhandl. d. Berl. Akad. 1863) 1864, 65 und Zeitschr. d. deutsch. geolog. Gesellsch. 1864, 16, 355-356. Krantz, Verh. d. naturhist. Ver. pr. Rheinl. u. Westf. 1865, 22, 19-20, Sitzungber.

**) Ann. d. k. k. naturhist. Hofmuseum in Wien $1891,6,131 \mathrm{ff}$.

***) Es bleibt dahin gestellt, ob das Magnesium der Metallegirung angebört, oder ob es selbst durch die schwache Säure dem etwa beigemengten Olivin entzogen worden ist. 
Die quantitative Analyse ergab:

$\begin{array}{ll}\text { Schwefeleisen } & \text { Spur } \\ \text { Graphit } & 0,025 \\ \text { Silicate } & 0,008 \\ \text { Phosphornickeleisen } & \mathbf{0 , 1 1 5} \\ \text { Phosphor } & \mathbf{0 , 0 7 2} \\ \text { Magnesium } & 0,033 \\ \text { Kupfer } & 0,127 \\ \text { Kobalt } & 0,701 \\ \text { Nickel } & 7,309 \\ \text { Eisen } & \mathbf{9 1 , 0 2 0} \text { (Mittel aus } 91,223 \text { u. 90,818) }\end{array}$

Bei der schon mebrfach gemachten Annahme, dass der Phosphor von ganz fein dem Nickeleisen beigemengtem, bekanntlich in kalter dunner Salzsäure immerhin etwas löslichem Phosphornickeleisen herrührt, besteht das Meteoreisen aus :

\begin{tabular}{lr} 
Nickeleisen & 98,812 \\
Phosphornickeleisen & 0,565 \\
Graphit & 0,025 \\
Schwefeleisen & Spur \\
Silicate & 0,008 \\
\cline { 2 - 2 } & 99,410
\end{tabular}

Dem Nickeleisen käme dann die Zusammensetzung zu:

\begin{tabular}{lrr} 
Magnesium & 0,034 & \multicolumn{1}{c}{ Mol.: } \\
Kupfer & 0,129 & \\
Kobalt & 0,709 \\
Nickel & 7,346 & \\
Eisen & 91,782 & $1,37=1$ \\
& 100,000 & $16,42=12$
\end{tabular}

Dieselbe entspricht beinahe der mittleren Zusammensetzung des Balkeneisens (Kamacit) 1:14; es treten mithin in dem Meteoreisen von Werchne Udinsk, wie das die Betrachtung der geätzten Schlifflächen schon zeigt, die nickelreicheren Tänitlamellen zwischen den breiten Balken sehr zurück. Sie sind so dünn und spärlich, dass sie sich beim Auflösen des Stuckes in der kalten verdunnten Säure schon völlig mit dem Kamacit aufgelöst haben, während sie sonst mehr oder minder angenagt zurückbleiben. 


\section{Die Silicate im Meteoreisen von Netschaëvo in Russland.}

Das 1846 bei Netschaëvo im Gouvernement Tula in Russland gefundene und durch J. Auerbach und W. Haidinge ${ }^{*}$ ) beschriebene Eisen zeichnet sich vor allem durch seine eckigen, von Haidinger für eingeschlossene Bruchstucke gebaltenen, bis wallnussgrossen Partion von Silicaten aus, welche übrigens auch in schlierenartigen Nestern und in einzelnen Körnern das Eisen durchsetzen.

Diese steinigen Nester sollen durch ihre Häufigkeit das Eisen manchmal wie eine Breccie erscheinen lassen, aber keine Störung des oktaëdrischschaligen Gefüges hervorrufen.

Aus einer Analyse berechnete Auerbach die mineralische Zusammensetzung dieser Nester zu :

$$
\begin{array}{cl}
72,98 \% & \text { Olivin } \\
16,70 & - \text { Nickeleisen (Co-haltig) } \\
10,21 & - \text { Plagioklas und Augit? } \\
0,11 & - \text { Chromit } \\
\text { Spur } & \text { Schwefeleisen } \\
\hline 100,00 &
\end{array}
$$

Ihr Volumgewicht fand Haidinger $=4,153$.

Von diesem werthvollen Eisen besitzt das hiesige Museum aus der Krant z'schen Sammlung ein $153 \mathrm{~g}$ schweres Stick, hier und da mit kleinen Silicatnestern, sowie einen $0,5 \mathrm{~g}$ schweren Splitter eines solchen.

Einen kleinen Theil des letzteren benutzte ich zu den folgenden Untersuchungen.

In kochender Salzsäure löst es sich nur theilweise auf unter Entbindung von Schwefelwasserstoff und Abscheidung steifer Kieselgallerte. In dieser Lösung konnten nur. Eisen, Magnesia, Spur Thonerde, Kalkerde und Nickel, kein Chrom (Daubréelith) aufgefunden werden.

Der lösliche Antheil besteht demnach aus etwas Nickeleisen, reichlich Schwefeleisen, viel Olivin und elwas Plagioklas.

Der in Flusssäure gelöste Lösungsrúckstand enthält viel Magnesia, wenig Eisen, etwas Thonerde und Kalkerde, Spur Natron, kein Kali. Das machte den Bestand aus Enstatit, vielleicht mit etwas Plagioklas wahrscheinlich.

*) W. Ha idinger, Sitzungsber. d. Wiener Akad., math.-nalurw. Cl. 1860, 42, 507-518; 1864, 49, 496. - Bull. d. l. soc. imperr. des naturalistes de Moscou 1860, 33, 362-376.

J. Auerbach, Bull. d. l. soc. imp. d. nat. de Moscou 1858, 31, 331-332; 1862, 35, 628-633. - Pogg. Ann. 1863, 118, 363-367. 
Unter dem Mikroskope betrachtet besteht der Lösungsrücksland aus kleinen Körnern oder Kornaggregaten und zwar uberall von ganz gleicher Beschaffenheit. Sie sind farblos oder zart grün angehaucht, zu schwach zur Erkennung eines etwaigen Pleochroismus. Man könnte sie leicht mit Quarz verwechseln, wenn nicht hier und da deutliche prismatische Spaltbarkeit zu erkennen wäre, welcher ausnahmslos die Schwingungsrichtung des Lichtes folgt.

Das ist entscheidend für En s tatit. Körner von Plagioklas und Quarz konnten nicht sicher, solche von Augit, Zirkon, Chromit bestimmt nicht aufgefunden werden.

Die Silicate in diesem Meteoreisen sind mithin sehr abweichend von denen im Toluca-Eisen, sie bestehen aus Enstatit und Olivin nahezu in gleichen Mengen untermischt mit etwas Nickel- und Schwefeleisen, sowie mit etwas Plagioklas. Ob auch Chondren in diesem körnigen Gemenge sich einstellen, konnte bei dem spärlichen Beobachtungsmateriale nicht festgestellt werden.

Das Eisen von Netschaèvo vermittelt mithin einerseits Olivin- und Bronzit-Pallasite mit einander, andererseits die Mesosiderite mit dem Meteoreisen.

\section{Snblimirte Mineralien vom Krufter-Ofen am Laachersee.}

Unter diesem Titel hat K. Busz im dritten Theile dieser Mittheilungen Nr. 19*) Eisenglanzkrystalle beschrieben, die mit kleinen röthlichen Krystallen besetzt waren, welche er damals als $\mathrm{R} u \mathrm{til}$ bestimmte.

Neuere Funde von dort haben ihm aber gezeigt, dass alle diese rothen Krystalle 0 li vin sind, mögen dieselben auf den Eisenglanzkrystallen aufgewachsen sein oder in den Drusen des basaltischen Gesteines neben den Eisenglanzen sich befinden**).

\section{Antimonnickel (Breithauptit) von St. Andreasberg im Harz.}

K. Busz fand 1893 im Museum trefflich glänzende Krystalle von Antimonnickel (Breithauptit) im Kalkspath von St. Andreasberg.

Er unterzog dieselben einer erneuten krystallographischen Untersuchung, da seit dem Jahre 1840, in welchem Breithaupt dieses Mineral krystallographisch untersucht hatte, an demselben keine weiteren Beobachtungen angestellt worden sind $\left.{ }^{* * *}\right)$.

*) Diese Zeitschr. 1891, 19, 24.

**) Sitzungsberichte der niederrheinischen Gesellschaft zu Bonn 1894, 33.

***) Ebenda $32 \mathrm{f}$. 
Die mil schwacher Säure aus dem Kalkspath herausgelösten Krystalle zeigen vorherrschend eine Pyramide, untergeordnet das hexagonale Prisma gleicher Stellung, die Basis und einmal eine Fläche einer steileren Pyramide der nämlichen Stellung.

Nimmt man das Antimonnickel isomorph mit Arsennickel (Rothnickelkies) und mit Schwefelnickel (Millerit, Beyrichit), folglich rhomboëdrischhemiëdrisch, so sind jene beobachteten Formen :

$$
\{33 \overline{6} 4\} \frac{3}{2} P 2,\{11 \overline{2} 0\} \infty P 2,\{0001\} 0 R,\{7.7 . \overline{1} 4.2\} 7 P 2 \text {. }
$$

Aus den Messungen der herrschenden Pyramide berechnete B u z z das Axenverhältniss :

$$
a: c=1: 0,9962 \text {. }
$$

Es wurde an den Krystallen auch eine Zwillingsbildung nach $\{33 \overline{6} 4\}{ }_{2}^{3} P 2$ beobacbtet.

Nimmt man dagegen mit B reithaupt das Antimonnickel isomorph mit Magnetkies als holoëdrisch-hexagonal, so zeigen diese Krystalle die Combination :

$$
\{30 \overline{3} 2\} \frac{3}{2} P,\{10 \overline{1} 0\} \infty P,\{0004\} 0 P,\{70 \overline{7} 1\} 7 P,
$$

und das Axenverhältniss $a: c$ ist $1: 0,8627$.

\section{Eisenhaltige Opale im Siebengebirge.}

Das Vorkommen von eisenhaltigen und deshalb gelben, grünen, braunen und braunrothen Opalen (Opaljaspis, Jaspopal) im Siebengebirge ist nach von Dechen ein sehr mannigfaltiges*).

Bald bildet er Gänge oder Kluftausfüllungen im Andesit (NW.-Abhang der Rosenau, Stenzelberg) oder im Trachyt (am Steinchen am SW.-Fusse des Drachenfels), bald Drusen- oder Kluftausfüllungen im Basalt (Obercassel, Limberg), ferner Gänge im Trachytconglomerat (Langenberg bei IIeisterbach, Casselerheide, Rosenau), sodann auch Drusen- und Kluftausfüllungen, sowie das Versteinerungsmittel von Pflanzenresten (Holzopal) in den tertiären Kieselconglomeraten (Obercassel, Wintermühlenhof (Quegstein), Muffendorf).

Nach meinen seit einigen Jahren an besonders schönen, neuen Aufschlüssen angestellten Beobachtungen hat sich der Opal namentlich bei der Verwitterung (Kaolinisirung) der Trachytconglomerate (Tuffe) gebildet, die im Tertiär unmittelbar ther den Kieselconglomeraten liegen und von den festen Trachyten, Andesiten und Basalten durchsetzt werden.

*) Geogn. Führer in das Siebengebirge am Rhein. Bonn 1861, 121, 158, 258, $264,268,27,2$. 
Durch die bei der Kaolinisirung der Feldspäthe abgeschiedene gelöste Kieselsäure scheinen die unter dem Trachyttuffe liegenden, bis dahin losen Quarzsande und Quarzgerölle zu Quarziten und Kieselconglomeraten verfestigt zu sein, und durch die Durchtränkung einzelner Partien des noch mehr oder weniger frischen Trachyttuffes oder der zahlreich in demselben eingeschlossenen bald grossen, bald kleinen Trachytbomben mit dieser Kieselsäure dürften die silicirten Trachyte entstanden sein, welche namentlich um die Rosenau vorkommen und so lange fur Liparite angesprochen worden sind ${ }^{*}$ ).

Wohl die schönsten und grössten Knollen von Opal finden sich auf der jelzt an mehreren Stellen im Siebengebirge gut aufgeschlossenen Grenze von Trachyttuff und festen Eruptivgesteinen z. B. am Ostgehänge des Stenzelberges.

Die von Pohlig geäusserte Ansicht, diese Knollen seien nicht Eisenopal, sondern ein "Opalobsidian (***), veranlasste W. Brubns***) solche von dort im hiesigen Museum niedergelegten Opale einer chemischen und mikroskopischen Untersuchung zu unterziehen.

Erstere ergab dasselbe wie die fruher von Schnabel, Bischof und von der Mark ausgeführten Analysen von Opalen des Siebengebirges.

In Kalilauge lösten sich $90,2 \%$, der in Salzsäure lösliche Rúckstand enthielt nur Eisen; der Wassergehalt betrug 4,47\% .

Der Opal der benachbarten Rosenau ergab bei gleicher Behandlung $95,8 \%$ löslich in Kalilauge und 4,4\% Wassergehalt.

Unter dem Mikroskope besteht der Stenzelberger Opal aus amorpher farbloser Masse, in welcher zahlreiche meist rundliche Anbäufungen brauner Eisenverbindungen liegen.

Der an und für sich farblose Opal umschliesst demnach wohl, wie v. Dechen schon vermuthet hatte, gleich dem gelben, Eisenkiesel, Brauneisenstein in ganz fein vertheiltem Zustande.

\section{Kupferglanzzwilling nach $\{130\} \infty \breve{P 3}$ von Bristol in Connecticut.}

\section{(E. Kaiser.)}

Unter den zahlreichen und schönen im Besitze des hiesigen mineralogischen Museums befindlichen Kupferglanzzwillingen und Drillingen dieses Fundortes, bei denen sich die Streifen auf $\{001\} 0 P$ unter fast $120^{\circ}$ an der Zwillingsfläche $\{110\} \infty P$ treffen, fand sich auch e in Zwilling, bei dem die Streifen etwa $60^{\circ}$ mit einander bildeten (vergl. nebenstehende Fig. 3, die

*) Verhandl. d. naturhist. Ver. pr. Rheinl. u. Westf. 1883, 40, 391-396 und Sitzungsber. d. niederrheinischen Gesellsch. z. Bonn 1885, 119-127.

**) Sitzungsber. d. niederrhein. Gesellsch. zu Bonn 1891, 70.

***) Ebenda 1893, 8 . 
in doppelter Grösse des Krystalles gezeichnet ist). Hier liegt mithin entweder ein Zwilling desselben Gesetzes vor, bei dem die Zusammenwachsungsfläche aber nicht die Zwillingsfläche $\{110\} \infty P$, sondern die Normalfläche zu dieser ist, also ein sogenannter Durchkreuzungszwilling nach $\infty P$, oder aber ein $Z$ willing nach $\{130\} \infty \breve{P} 3$, wie solche z. B. beim Gerussit bekannt geworden sind *).

Im ersteren Falle müssen die Prismenflächen $n^{\prime}$ und $\underline{m^{\prime}}$ zusammenfallen, im letzteren Falle einen ganz stumpfen einspringenden Winkel einschliessen. Durch die Messungen im Fernrohrgoniometer konnte nun mit voller Sicherheit nachgewiesen werden, dass die Prismenflächen $m, m^{\prime}, \underline{m}^{\prime}, \underline{m}$, genau in derselben Zone liegen und dass

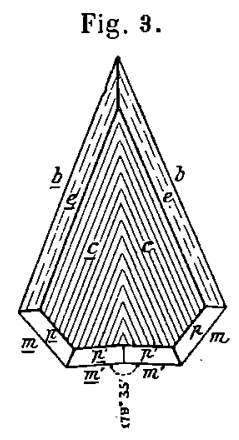
die Flächen $m^{\prime}$ und $\underline{m}^{\prime}$ einen Normalenwinkel von $0025^{\prime}$ einschliessen.

Die gemessenen Winkel betrugen:

$$
\begin{aligned}
& \left.\begin{array}{l}
m: m^{\prime}=(1 T 0):(110)=60^{\circ} 13^{\prime} 0^{\prime \prime} \\
m: m^{\prime}=(110):(110)=601130
\end{array}\right\} \text { Mitlel } 60^{0} 12^{\prime} 15^{\prime \prime} \\
& m^{\prime}: \underline{m}^{\prime}=(110):(1 T 0)=025 \\
& m: \underline{m}=(1 T 0):(T 10)=120 \quad 7
\end{aligned}
$$

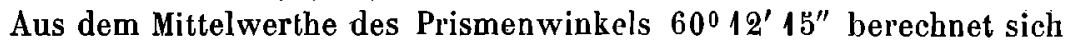
der einspringende Winkel an der angenommenen Zwillingsgrenze $\{130\}$ $\infty \breve{P} 3$ zu $0^{0} 24^{\prime} 29^{\prime \prime}$.

Aus dem Vergleiche des gemessenen und berechneten Winkels ergiebt sich, dass die Annabme einer Zwillingsbildung nach $\infty \breve{P} 3$ voll berechtigt ist.

Der gemessene Prismenwinkel $\{110\} \infty P$ weicht von dem gewöhnlich angegebenen Werthe von $60025^{\prime}$ erheblich ab**). Bestäligt wurde diese Abweichung noch durch Messungen an gut ausgebildeten Krystallen desselben Fundortes. Diese ergaben an Krystall Nr. $160^{\circ} 18^{\prime} 30^{\prime \prime}$, Nr. $260^{\circ} 15^{\prime}$, Nr. 3 $60^{\circ}$ 13'. Aehnliche Abweichungen hat schon B o e $r$ is angegeben **).

Messungen in einer anderen als der Verticalzone konnten wegen der starken Streifung nach der Brachydiagonale mit Sicherheit nicht vorgenommen werden.

Das Axenverhältniss des Kupferglanzes unterliegt demnach nicht unbeträchtlichen, vermuthlich in der chemischen Zusammensetzung begrundeten Schwankungen.

Es sind mithin am Kupferglanz jetzt Zwillinge nach $\{110\} \infty P$, $\left.\{112\}_{\frac{1}{2}} P,\{043\} \frac{4}{3} \breve{P}_{\infty}^{* * *}\right),\{011\} \breve{P} \infty$ und $\{130\} \infty \breve{P} 3$ bekannt.

*) Pogg. Ann. 1875, 156, 558.

**) Diese Zeitschr. 1894, 23, 238.

***) oder $\{032\} \frac{3}{2} \breve{P} \infty$, wie B o eris, diese Zeitscbr. 1894, 23, 238 angiebt. 